\title{
Comparison of two Meglumine- Diatrizoate based bowel preparations for computed tomography colonography: comparison of patient symptoms and bowel preparation quality
}

\section{Abstract}

Introduction: To investigate the impact of two Meglumine-Diatrizoate based bowel preparation regimes for computed tomography colonography (CTC) on the patient experience and image quality.

Methods: 100 patients consumed Meglumine-Diatrizoate at 24 hours and 12 hours prior to the CTC examination. 50 patients followed regime 1 (50:50), $50 \mathrm{ml}$ of Meglumine-Diatrizoate at both 24 and 12 hours prior to the examination. 50 patients followed regime 2 (75:25), $75 \mathrm{ml}$ of MeglumineDiatrizoate at 24 hours prior to the examination and $25 \mathrm{ml}$ of Meglumine-Diatrizoate at 12 hours prior to the examination. All patients completed a questionnaire to indicate the time of onset of adverse effects and when they were most severe. Five advanced practitioners assessed the image quality in a visual grading study. Visual grading characteristic (VGC) analysis was applied with regime 1 as the reference condition and regime 2 and test condition; test alpha was set at 0.05 .

Results: Image quality was assessed with successful bowel cleansing as the scoring criteria for the visual grading study. The bowel cleansing as provided by the two Meglumine-Diatrizoate regimes was revealed not to be statistically different, with the area under the VGC curve and $95 \%$ confidence intervals $0.487(0.287,0.701), p=0.887$. Patients taking the $75: 25$ bowel preparation experienced a shorter median time to the onset of adverse effects.

Conclusion: There was no observed difference in Image quality criteria score for the two MeglumineDiatrizoate based bowel preparation with more predictable adverse effects of MeglumineDiatrizoate with the 75:25 preparation.

Implications for Practice: Providing patients with a higher contrast burden 24 hours prior to CTC may have a positive impact on the patient experience without compromising image quality. 


\section{Introduction}

It is essential that computed tomography colonography (CTC) achieves maximal diagnostic performance to enable the detection of cancer and pre-cancerous growths (polyps). Recent studies suggest variable performance in the technique, with sensitivity and specificity quoted in the range of 76-91 \% and 81-99 \% respectively..$^{1,2} \mathrm{~A}$ recent systematic review ${ }^{3}$ revealed that preparation regime, CTC imaging technique and image evaluation methods were all variable and it is not unreasonable to expect variation in performance as a result of these factors, though they were not specifically investigated in this recent review.

CTC imaging technique (parameters, position) and image evaluation can be modified with minimal or no impact on the experience of the patient during the examination. However, a suitable lowresidue diet and bowel preparation has a huge impact on the success of the procedure and the correct balance between image quality, the detection of intra-colonic pathology and the physiological impact on the patient must be considered. Successful bowel preparation requires residual fluid and faeces to be 'tagged' by an iodinated contrast agent, with the desired outcome of achieving a homogenous residual fluid with a density of 900-1000 Hounsfield Units (Hu)..$^{3-5}$ This allows differentiation of pathology $(200 \mathrm{Hu})$ and residual faecal matter $(100 \mathrm{Hu})$ from residual colonic fluid ( $950 \mathrm{Hu})$. Residual fluid above $1000 \mathrm{Hu}$ risk being degraded by beam hardening artefact especially at low kVP levels. Fluid with comparable HU densities can mask pathology that is semi-submerged in fluid, leading to false negatives and decreasing the sensitivity of the CTC procedure. ${ }^{6,7}$

Colonic lavage remains a significant issue within CTC as patients must cope with restricted food consumption and loose stools prior to their examination. ${ }^{8}$ There is currently no standard protocol for optimum bowel preparation in the USA, Britain or Europe. Limited advice from the European Society of Gastro-intestinal and Abdominal Radiology (ESGAR) and the American College of Radiology (ACR) has led to protocol derivation from barium enema and optical colonoscopy procedures, with the addition of faecal/fluid tagging. This results in a wide variety of bowel preparation protocols, that are dependent on previous CTC experience, peer review guidance and contractual agreements. ${ }^{9}$

Meglumine-Diatrizoate (Gastrografin ${ }^{\circledR}$; Bayer Schering Pharma, Berlin, Germany) is an oral and rectal ionic contrast agent, historically used in the treatment of paediatric bowel obstruction, since the formula causes colonic lavage. Lavage is caused by Tween 80 (Polysorbate 80), an oily yellow fat that is not broken down or absorbed by the large bowel and is present in a concentration of $0.75 \%{ }^{10}$ The differential density and osmolality of the contrast compared to the blood plasma causes a shift of fluid by osmosis from the blood capillaries in the mucosa into the bowel lumen. ${ }^{11}$ Tween 80 also acts as a stool emulsifier and further increases the contractility of smooth muscles. This facilitates the 
movement of bowel contents through the comparatively narrowed lumen to the rectum for excretion.

Meglumine-Diatrizoate based preparations satisfy the four main criteria for CTC bowel preparation:

- A small risk of anaphylactoid reaction or renal damage; ${ }^{4}$

- A simple preparation resulting in good compliance, ${ }^{12}$

- A small amount of residual faeces, thus reducing interpretation error ${ }_{i}^{13}$

- Residual fluid homogeneity is good (900-1000 HU). ${ }^{14}$

The density and homogeneity of residual colonic fluid is pivotal to the sensitivity of the test. ${ }^{15}$ Studies with residual fluid below $500 \mathrm{HU}$ can lead to false positives and studies with residual fluid above 1400 HU can lead to beam hardening artefacts, increased radiation dose, and loss of image resolution. ${ }^{5}$

CTC preparation protocols using Meglumine-Diatrizoate have been developed using national and international guidance. ${ }^{16,17}$ In our institution, a split-dose protocol consisting of $50 \mathrm{ml}$ of MeglumineDiatrizoate 24 hours and 12 hours prior to the procedure was introduced as part of a Radiographer led service that performs examinations on approximately 1200 CTC patients per annum. Regular audit against the findings from institutional endoscopy and a regional cancer registry has ensured that the diagnostic performance of this test has been suitable.

This study describes the impact of a change in bowel preparation protocol on image quality and patient symptoms.

\section{Materials and Methods}

The local Research and Development department considered the modification of bowel preparation to be an evaluation of service provision. All patients attending for CTC provide written consent for their data to be used in research and service improvement; this is standard procedure and was not introduced for this study. Data were collected on consecutive patients between December 2017 and January 2018. If patients withdrew consent or failed to comply with the preparation regime, they were excluded from the study. From January 2018, the CTC preparation in our institution changed due to observations made by the Advanced Practitioner Radiographers and results gained from an annual audit of the patient experience. Patients indicated the need to defecate in the early hours of the morning prior to the test and this was an unwanted scenario. In an attempt to improve test tolerance and bowel preparation we made a change to the protocol with the intention of stimulating increased lavage in the earlier stages of the protocol. 


\section{Patient Groups and Bowel Preparation}

Patient group 1, examined in December 2017, consisted of 50 patients. The preparation regime required the cohort to have a 50:50 split dose preparation, taking $50 \mathrm{ml}$ of Meglumine-Diatrizoate at 12 and 24 hours prior to the CTC examination. Patient group 2, examined in January2018, consisted of 50 patients. The preparation regime required the cohort to have a 75:25 split dose preparation, taking $75 \mathrm{ml}$ of Meglumine-Diatrizoate at 24 hours prior to the CTC examination and $25 \mathrm{ml}$ at 12 hours prior to the examination. For both groups, the patients were instructed to take the 24 -hour preparation volume at $7 \mathrm{AM}$ the day before the test and the 12-hour preparation volume at $7 \mathrm{PM}$ the day before the test. All CTC procedures occurred between 7:30-9:15 AM. On the morning of the CTC procedure, patients completed a questionnaire to determine compliance and the impact of the bowel preparation, Table 1. Patient demographics, age and sex, were recorded for both groups.

\begin{tabular}{|l|c|}
\hline Question & Response Option \\
\hline Did you take the correct volume of preparation at the correct time? & Yes / No \\
\hline $\begin{array}{l}\text { Did you experience diarrhoea after taking the preparation? } \\
\text { If yes, at what time did this start? } \\
\text { If yes, at what time were the effects most severe? }\end{array}$ & Yes / No \\
\hline Did you wake in the night because of the preparation? & Yes / No \\
\hline $\begin{array}{l}\text { On a scale of 1-10 (severe to not severe) how would you rate the effect of the } \\
\text { preparation? }\end{array}$ & (Severe) 1-10 (Not Severe) \\
\hline $\begin{array}{l}\text { Have you taken this, or any other bowel preparation before? } \\
\text { If yes, what was the name of the preparation? }\end{array}$ & Yes / No \\
\hline $\begin{array}{l}\text { How does this bowel preparation compare to the previous bowel } \\
\text { preparation? }\end{array}$ & Worse / Same / Better \\
\hline
\end{tabular}

Table 1: The questionnaire given to all patients as part of the CTC examination. 


\section{CTC Imaging Protocol}

Each patient was required to have a fully prepared colon for the examination. 20 milligrams in $1 \mathrm{ml}$ of subcutaneous Hyoscine $\mathrm{N}$ butyl-bromide, (Buscopan) was administered intravenously immediately before colonic tube insertion unless contraindicated by details extracted in the consent form (i.e. narrow angle glaucoma, myasthenia gravis, a previous allergy to hyoscine or atropinics (atropine, scopolamine), tachycardia, the patient has toxic megacolon (enlarged colon) or if they are male and have had obstructive prostatic hypertrophy). ${ }^{18}$ Glucagon Hydrochloride was not injected to aid colonic distension for patients that were contraindicated for Hyoscine $\mathrm{N}$ butyl-bromide injection as it is not currently recommended within UK practice. ${ }^{16}$ It is noted that Dyde et $a{ }^{16}$ do not recommend asking all patients if they suffer from glaucoma, rather instruct them to seek medical help if they develop painful blurred vision but at the time of study it was in our local protocol to restrict buscopan for patients in this group.

Colonic insufflation was obtained with an automated carbon dioxide insufflator $\left(\mathrm{PROTOCO}_{2} \mathrm{~L}, \mathrm{E}-\mathrm{Z}-\right.$ EM., Bracco, Milano, Italy). Manual insufflation with room air was not considered as it is not recommended by British/European guidance. ${ }^{16,19}$ The patient initially lay on their left side to obtain right hemi colon distension and the patient was scanned in the supine position before a further secondary position (prone or decubitus). The CTC was acquired with a $64 \times$ multi-detector CT scanner (Aquilion, Toshiba Medical Systems, Tokyo, Japan). Scans were obtained through the abdomen and pelvis using the following parameters: $120 \mathrm{kV} ; 200-400 \mathrm{~mA}$ with automatic exposure control; 64 rows $\times 0.5 \mathrm{~mm}$ collimation; and helical pitch, 53 (pitch factor 0.828 ). All images were reconstructed with a $0.5 \mathrm{~mm}$ effective thickness at $0.5 \mathrm{~mm}$ intervals. Patients did not receive an intravenous bolus injection of intravenous contrast, as this is not standard practice within our institution.

\section{Assessment of Image Quality}

CTC Image quality was assessed by 5 advanced CTC reporting practitioners with over 6 years' experience (minimum 600 cases) in providing a formal written report of the intraluminal component of the CTC examinations. Images were reviewed over multiple sessions with each case taking approximately 20 minutes to review; breaks were permitted and no time restriction was enforced. For the purpose of this study, the ordinal data was collected as part of a visual grading analysis (VGA) study, where the quality of bowel preparation (judged on the volume of residual faeces) grades were given the values of 1-4 respectively (higher value for better preparation). Evaluations of bowel preparation quality, based on the CTC images, were performed on a part 14 DICOM compliant EIZO RadiForce RX240 LED Monitor (2mpx, 1200x1600, contrast 1200:1, brightness $760 \mathrm{~cd} / \mathrm{m}^{2}$; Eizo, Hakusan, Japan) under low ambient lighting conditions. 
Figure 1 shows a typical example of the images reviewed, with the contrast density identified in the transverse colon for the 50:50 (a) and 75:25 (b) preparation regimes.
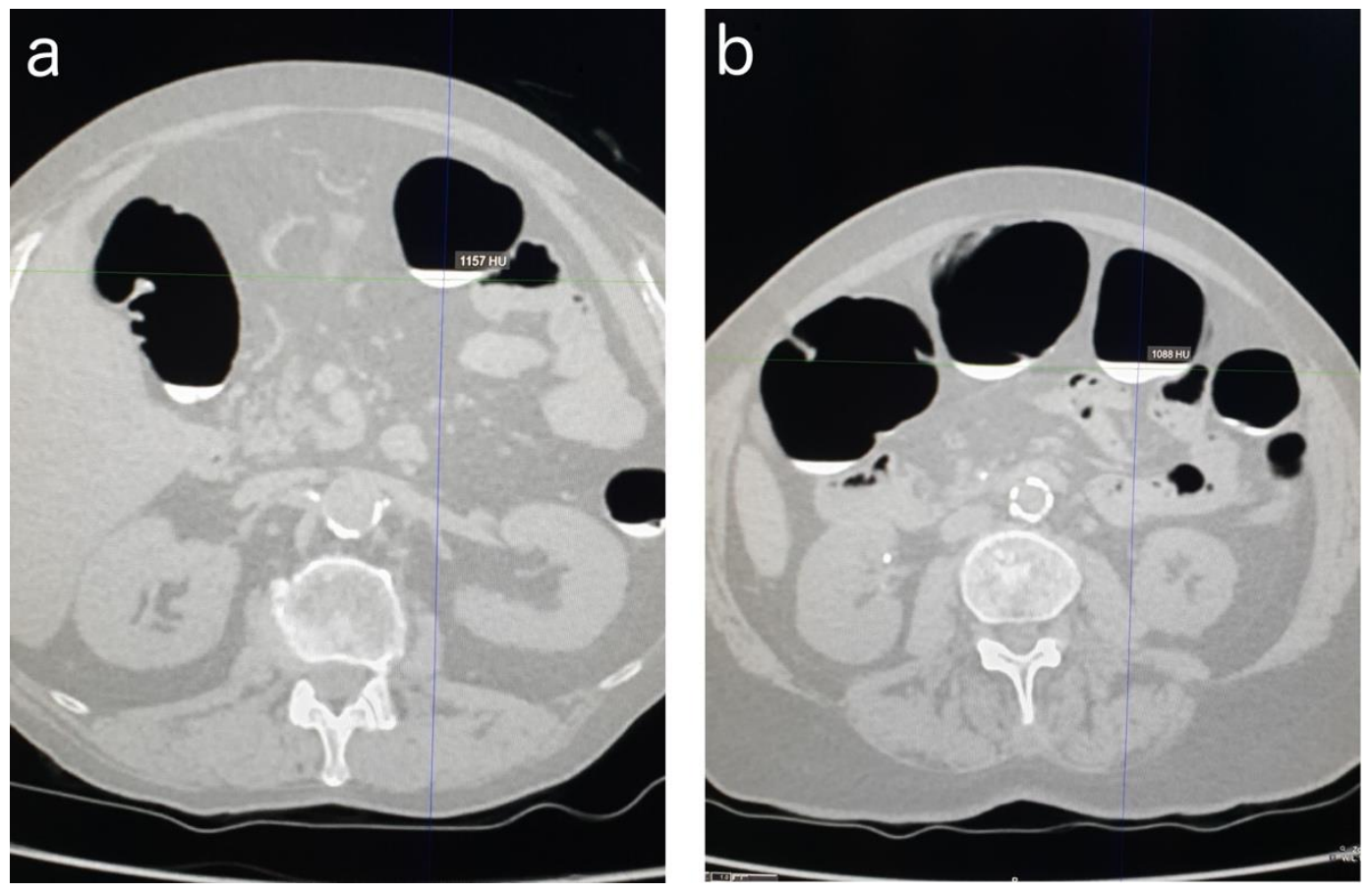

Figure 1: Tagged residual fluid in the transverse colon of two patients. An enhancement of $1157 \mathrm{HU}$ for the 50:50 bowel preparation (a), and an enhancement of $1088 \mathrm{HU}$ in for the 75:25 bowel preparation (b).

\section{Statistical Analysis}

Data recorded from the VGA study were analysed using VGA Analyser Software ( $V_{1}$.0, release 2). ${ }^{20,21}$ This produces the area under the visual grading characteristic curve ( $A \cup C_{V G C}$ ) and asymmetric $95 \%$ confidence intervals for the comparison of image quality between the 50-50 preparation and the 7525 preparation. The reference condition was the 50-50 preparation. For a difference in image quality criteria score (ICS) to be considered significantly different, the $95 \%$ confidence interval must not include 0.5 and the $p$-value must be less than $0.05 \cdot 22,23$

A chi-squared test, from a $2 \times 2$ frequency table (male:female), was used to test equal distribution of male and females in the patient groups and an unpaired t-test was used to look for a difference in the mean age of each patient group. For both tests, test alpha was set at 0.05 . 


\section{Results}

\section{Patient Groups \& Demographics}

100 patients took part in this study; no patients were excluded from the study for failing to follow the preparation regime. The demographic information of the two patient groups (50-50 and 75-25) preparation is displayed in Table 2. A two-tailed Chi-squared test indicated that there was no difference in the groups based on gender $\left(\chi^{2}=0.644, p=0.422\right)$. An unpaired t-test revealed no statistical difference between groups based on age (mean difference and 95\% confidence interval, $0.24(-3.53,4.01), p=0.900)$. Since the populations are not considered to be statistically different, we have assumed that any difference in symptoms experienced by the patients have been caused by the difference in preparation regime.

\begin{tabular}{|l|c|c|}
\hline Criteria & Group 1 (50-50) & Group 2 (75-25) \\
\hline Male & 25 & 29 \\
\hline Female & 25 & 21 \\
\hline Age Range & $50-95$ & $43-94$ \\
\hline Age Mean + SD & $76.3 \pm 8.6$ & $76.0 \pm 10.3$ \\
\hline
\end{tabular}

Table 2: Demographic information for the two groups of patients.

\section{Patient Group 1 (50-50 Preparation)}

All patients stated that they had correctly followed the preparation regime, taking $50 \mathrm{ml}$ of Meglumine-Diatrizoate at $7 \mathrm{AM}$ and another $50 \mathrm{ml}$ at 7 PM the day before the CTC examination. All 50 patients in this group experienced diarrhoea and the mean start time was 10 AM, 3 hours into the 24-hour preparation. Patients reported the worst symptoms to occur at a mean time of 1 PM, 6 hours after the 24 -hour preparation. However, it is important to point out that 9/50 patients in this group experienced the most severe symptoms of diarrhoea at 10 PM, 15 hours after the 7 AM preparation and 3 hours after the 7 PM preparation.

$17 / 50(34 \%)$ of patients had taken a previous bowel preparation for another investigation. The distribution of these is described in Table 3. Of these patients, those that had previously taken Citrafleet considered the experience equal with the 50:50 preparation, those who had taken Citramag considered the 50:50 preparation better, those who had taken another Meglumine-Diatrizoate preparation considered the 50:50 preparation to be better or equal to the previous preparation. Only 4 patients considered the 50:50 preparation to be worse than a previous bowel preparation; they had taken Movicol (3) and Picolax (1). All remaining patients taking Movicol and Picolax found the 50:50 preparation to be better. 


\section{Patient Group 2 (75-25 Preparation)}

Again, all patients stated that they had correctly followed the preparation regime, taking $75 \mathrm{ml}$ of Meglumine-Diatrizoate at $7 \mathrm{AM}$ and another $25 \mathrm{ml}$ at $7 \mathrm{PM}$ the day before the CTC examination. All 50 patients in this group experienced diarrhoea and the mean start time was 9 AM, 2 hours into the 24 -hour preparation. Patients reported the worst symptoms to occur at a mean time of 11 AM, 4 hours after the 24 -hour preparation. In contrast to the 50-50 preparation, only 1 patient experienced the most severe symptoms of diarrhoea after taking the 12 -hour preparation, at 8 PM. This is evidence of the larger initial Meglumine-Diatrizoate loading bringing diarrhoea on earlier in the daytime; a graphical representation of these data can be found in Figure 2 and 3 .

$18 / 50(36 \%)$ of patients had taken a previous bowel preparation for another investigation. The distribution of these is described in Table 3. Of these patients, those that had previously taken Citrafleet considered the experience better or equal with the 75:25 preparation, those who had taken Citramag considered the 75:25 preparation to be either worse or equal, those who had taken another Meglumine-Diatrizoate (7) and MagCitrate (2) preparations considered the 75:25 preparation to be or equal to the previous preparation. 4 patients had previously taken Picolax with 1 considering the 75:25 preparation to be better, 1 equal, and 2 considered it to be worse. The remaining patient had taken Moviprep and considered the 75:25 preparation to be better.

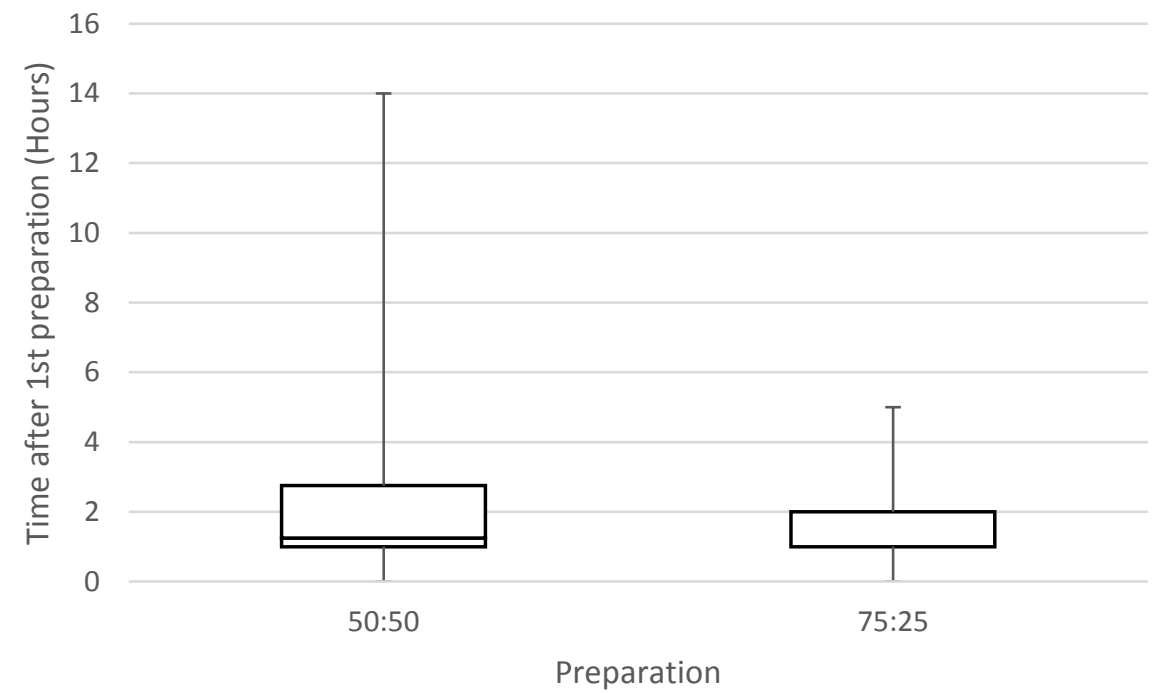

Figure 2: Time to onset of colonic lavage after first (24-hour) preparation volume for 50-50 and 75-25 preparations. The mean time for the 50-50 preparation was $2.8 \pm 3.3$ hours and for the 75:25 was 1.8 \pm 1.0 hours. 


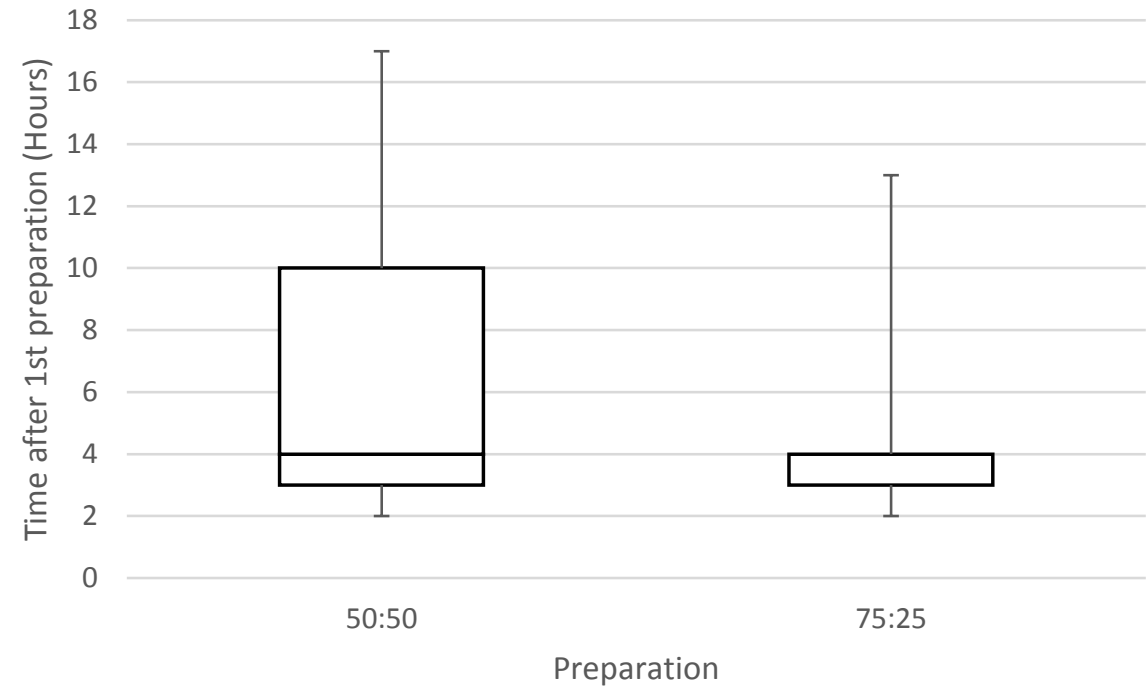

Figure 3: Time to most severe symptoms of diarrhoea after first (24-hour) preparation volume for 50-50 and 75-25 preparations. The mean time for the 50:50 preparation was 6.4 \pm 4.9 hours and for the 75-25 preparation was $3.9 \pm 2.0$ hours.

\begin{tabular}{|l|c|c|}
\hline Previous Preparation & $\begin{array}{c}\text { Group 1 (50-50) } \\
n\end{array}$ & $\begin{array}{c}\text { Group 2 (75-25) } \\
n \\
\text { (better/equal/worse) }\end{array}$ \\
(better/equal/worse)
\end{tabular}

Table 3: Summary of previous preparations taken by patients in both patient groups. ' $n$ ' refers to the number of patients who had taken each preparation for a previous procedure and the numbers in parentheses identify whether the patient considered the Meglumine-Diatrizoate preparation (50-50 or 75-25) to be better/equal/worse than the previous preparation. 


\section{Severity of Diarrhoea}

A Kolmogorov-Smirnov test of normality showed that the distribution of responses for the rating scale of diarrhoea severity did not significantly deviate from a normal distribution for patient group 1 $(D=0.13, p=0.319)$ or patient group $2(D=0.15, p=0.202)$. Despite normality, a non-parametric test was used to evaluate this ordinal data. A Mann-Whitney U Test for independent samples showed that the severity of diarrhoea was statistically worse for the $75-25$ preparation $(z=2.30, p=0.021)$. Despite increased severity of diarrhoea with the 75-25 preparation, fewer patients reported that they had been awake in the night in comparison to those who had taken the $50-50$ preparation (10\% versus $32 \%$ patients).

\section{Visual Grading Study - Image Quality}

5 advanced practitioner radiographers with a CTC speciality reviewed the images for patients in both groups. There was a minimum of 6 years of experience and each had conducted a minimum of 600 cases. They provided a rating of bowel preparation quality for each patient on a scale of 1-4, with the higher rating associated with better preparation. The area under the visual grading characteristic curve ( $A \cup C_{V G C}$ ) (Figure 4 ) was used as the figure of merit (FOM) when comparing the image quality criteria score (ICS) for the quality of bowel preparation between the 75-25 and the 50-50 bowel preparations. If the curve sits in the lower portion $\left(A \cup C_{V G C}<0.5\right)$ there is a preference for the 50-50 preparation and if it sits in the upper portion ( $A \cup C_{V G C}>0.5$ ) there is a preference for the $75-25$ preparation. In this instance the $A U C_{V G C}(95 \% \mathrm{Cl})$ was $0.487(0.287,0.701)$. Since the $95 \%$ confidence interval includes 0.5 , the difference in ICS is not considered to be statistically significant $(p=0.887)$. Therefore, the image quality provided by the two bowel preparations can be considered to be approximately equal. 


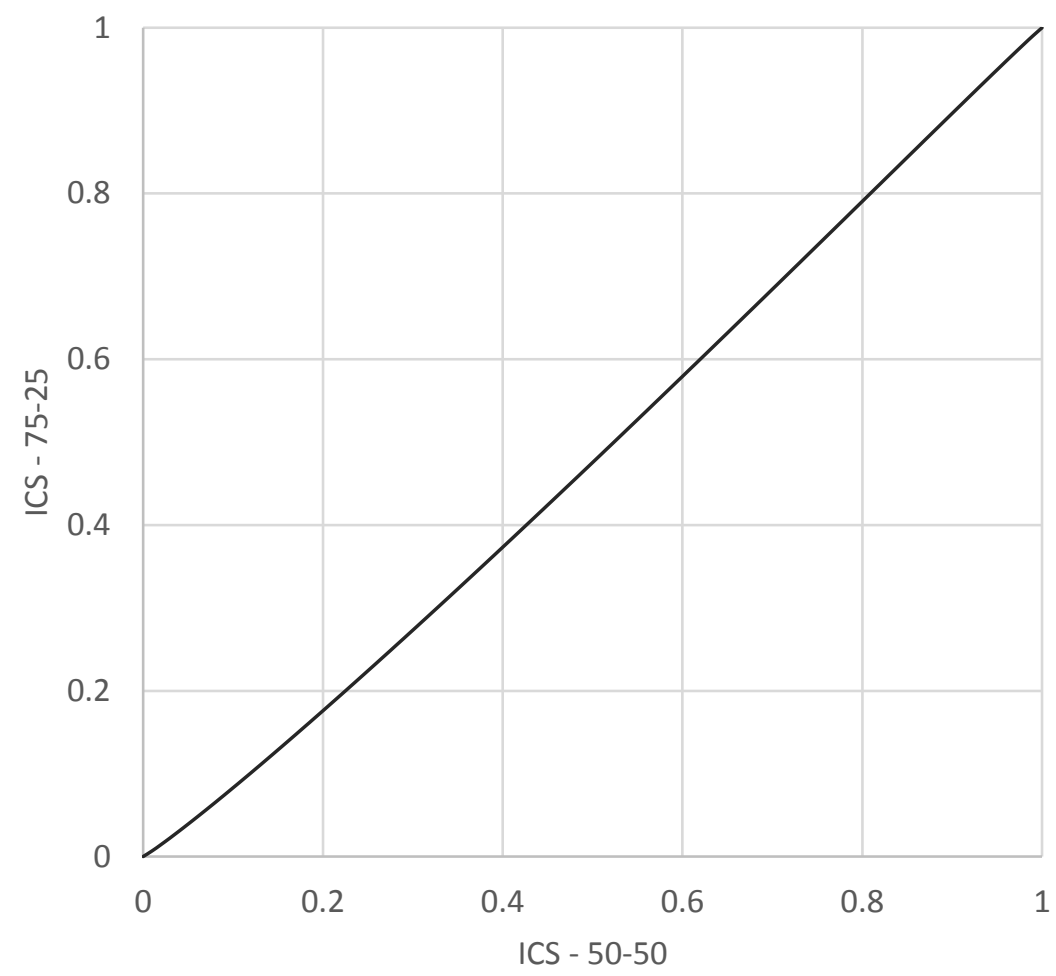

Figure 4: The VGC curve for the comparison of ICS between preparations using 50-50 and 75-25 preparation regimes. The AUCVGC and 95\% confidence intervals are $0.487(0.287,0.701)$ - the difference was not statistically significant.

\section{Discussion}

The aim of this study was to complete a comparison of two Meglumine-Diatrizoate based bowel preparations, accounting for the image quality provided by each preparation regime and the impact of each bowel preparation regime on the patient. We proposed that a bowel preparation with a higher iodine loading 24-hours prior to the CTC procedure and a lower iodine loading 12-hours prior to the procedure $(75-25)$ in comparison to a conventional bowel preparation regime that equally split the iodine load at 24- and 12-hours prior to the procedure (50-50) would provide favourable results. The important outcome from the study is that image quality was maintained and considered approximately equal when using the 75-25 preparation in place of a 50-50 preparation.

All patients involved in this study completed the preparation to a satisfactory standard for the examination to be completed. Typically, this institution has only a $2 \%$ failure rate in terms of bowel preparation, so this outcome is not unexpected. The patients involved in this study were asked to provide a basic report on the symptoms they experienced and given the similarity between the 
groups in terms of age and gender and the fact that they were consecutive symptomatic patients, the differences experienced are assumed to be due to the quantity of the Meglumine-Diatrizoate ingested at 24 - and 12 -hours prior to the procedure.

The patients completing the 75-25 preparation experienced a greater severity of symptoms of diarrhoea than those completing the 50-50 preparation. This is perhaps not unexpected considering the $50 \%$ increase in the volume of Meglumine-Diatrizoate 24 -hours prior to the procedure which is likely to cause a stronger osmotic effect. While the symptoms were judged to be worse by this unpaired sample of patients there is a potential improvement in the overall experience for the patient as the onset of symptoms was more predictable in terms of the timeframe for the symptoms to begin and for the symptoms to be at their most severe. This could be useful information to help inform the expectations of the patients prior to taking the preparation. Another aspect that may be considered important to patients is the improved likelihood of the worst symptoms to be experienced during a more predictable range of normal waking hours. Typically, the patients taking the $75: 25$ preparation experienced the worst symptoms $3-4$ hours after the 24 -hour preparation, compared to a larger range of 3-10 hours after the 24 -hour preparation the 50:50 preparation. In addition, only 5/50 patients (10 $\%$ ) were woken in the night with symptoms when taking the $75: 25$ preparation compared to $16 / 50$ (32 $\%)$ for the 50:50 protocol.

The overall goal of any preparation regime is to provide good bowel clearance and tagging of residual fluid. This study confirms that Meglumine-Diatrizoate (in conjunction with a low residue diet), can provide adequate colonic lavage and faecal/fluid tagging within a $24 \mathrm{hr}$ protocol. The timing and iodine loading of the preparation has be shown to influence the severity and timing of symptoms in this study but in general the overall impact is low since the procedure only dictates that a single day is compromised with bowel preparation and restricted diet.

Previous research has concluded that other preparation regimes using magnesium citrate, Picolax, Citrafleet and lohexol may be an alternative to Meglumine-Diatrizoate as they are more palatable and cheaper than Meglumine-Diatrizoate, ${ }^{24,25}$ but a Meglumine-Diatrizoate preparation has been shown to provide consistently well prepared bowels with high density residual fluid that has high homogeneity and low viscosity. ${ }^{17}$ Optimum preparation is achieved with simple instructions that can be followed by the majority of the patients resulting in the high compliance that we have experienced in this study. Our attempted comparison to other bowel preparations is inconclusive and this is likely to be due to the variation in preparation regimes and the inability of patients to have a good recollection of medical history, ${ }^{26}$ particularly when they are anxious, which they are likely to 
experience when presenting for a CTC examination. More work is required to fully understand the impact of alternative preparation on bowel cleansing and the patient experience.

\section{Limitations \& Future Studies}

One of the important limitations to recognise is the questionnaire provided to the patients. To improve this for future studies we would ask the patients to be more specific about their symptoms and recognise cramps, pain and bloating as important in addition to diarrhoea. It would also have been valuable to measure how the different preparations had affected their daily routine and we also acknowledge that the comparison to other preparation is crude and open to subjectivity.

For future studies it may also be worthwhile investigating the impact of different bowel preparation in the six different regions of the bowel (caecum, ascending, transverse, descending, sigmoid, rectum). We propose to do this by assessing the CT Hounsfield Unit (HU) values of the residual fluid in each of these regions for different preparation regimes to provide a quantitative assessment of preparation quality.

\section{Conclusion}

Image quality was maintained when using a 75-25 bowel preparation regime, suggesting efficacy of this alternative. In addition, the symptoms of diarrhoea experienced by the patient were less likely to occur during the night time and overall were more predictable in terms of timing, which may provide useful information to help patients manage their expectations of the preparation, despite the severity of symptoms being worse than the 50-50 regime.

\section{Implications for Practice}

Providing patients with a higher contrast burden 24 hours prior to CTC may have a positive impact on the patient experience without compromising image quality. This evidence could be used to help manage the expectations of the patients in relation to the preparation regime and has the potential to reduce the risk of a disturbed night prior to the CTC procedure.

\section{References}

1. Lauridsen C, Lefere P, Gerke O, Hageman S, Karstoft J, Gryspeerdt S. Comparison of the diagnostic performance of CT colonography interpreted by radiologists and radiographers. Insights Imaging. 2013;4(4):491-497. doi:10.1007/\$13244-013-0260-x

2. Utano K, Nagata K, Honda T, et al. Diagnostic Performance and Patient Acceptance of 
Reduced-Laxative CT Colonography for the Detection of Polypoid and Non-Polypoid Neoplasms: A Multicenter Prospective Trial. Radiology. 2017;282(2):399-407. doi:10.1148/radiol.2016160320

3. Pickhardt PJ, Correale L, Delsanto S, Regge D, Hassan C. Ct colonography performance for the detection of polyps and cancer in adults $\geq 65$ years old: Systematic review and metaanalysis. Am J Roentgenol. 2018;211(1):40-51

4. Chang KJ, Kim DH. CTC technique: methods to ensure an optimal exam. Abdom Radiol. 2018;43(3):523-38 doi:10.1007/s00261-018-1499-y

5. Scalise P, Mantarro A, Pancrazi F, Neri E. Computed tomography colonography for the practicing radiologist: A review of current recommendations on methodology and clinical indications. World J Radiol. 2016;28;8(5):472-83 doi:10.4329/wjr.v8.i5.472

6. Barrett JF, Keat N. Artifacts in CT: Recognition and Avoidance. RadioGraphics. 2004;24(6):1679-91 doi:10.1148/rg.246045065

7. Mang T, Graser A, Schima W, Maier A. CT colonography: Techniques, indications, findings. Eur J Radiol. 2007;61(3):388-99 doi:10.1016/j.ejrad.2006.11.019

8. Thomeer $M$, Bielen $D$, Vanbeckevoort $D$, et al. Patient acceptance for CT colonography: What is the real issue? Eur Radiol. 2002;12(6):1410-5 doi:10.1007/s003300101082

9. Neri E, Halligan S, Hellström M, et al. The second ESGAR consensus statement on CT colonography. Eur Radiol. 2013;23(3):720-9 doi:10.1007/s00330-012-2632-x

10. Wood BP, Katzberg RW. Tween 8o/diatrizoate enemas in bowel obstruction. Am J Roentgenol. 1978;130:747-50 doi:10.2214/ajr.130.4.747

11. Rowe MI, Seagram G, Weinberger M. Gastrografin-induced hypertonicity. The pathogenesis of a neonatal hazard. Am J Surg. 1973;125(2):185-8 doi:10.1016/0002-9610(73)90025-1

12. Nagata K, Okawa T, Honma A, Endo S, Kudo S ei, Yoshida H. Full-laxative Versus Minimumlaxative Fecal-tagging CT Colonography Using 64-detector Row CT. Prospective Blinded Comparison of Diagnostic Performance, Tagging Quality, and Patient Acceptance. Acad Radiol. 2009;16(7):780-9 doi:10.1016/j.acra.2008.12.027

13. Slater A, Planner A, Bungay HK, Bose P, Milburn S. Three-day regimen improves faecal tagging for minimal preparation CT examination of the colon. Br J Radiol. 2009;82(979):5458 doi:10.1259/bjr/82959871 
14. Theis J, Kim DH, Lubner MG, del Rio AM, Pickhardt PJ. CT colonography after incomplete optical colonoscopy: bowel preparation quality at same-day vs. deferred examination.

Abdom Radiol. 2016;41(1):10-8 doi:10.1007/s00261-015-0595-5

15. Halligan S, Altman DG, Taylor SA, et al. CT Colonography in the Detection of Colorectal Polyps and Cancer: Systematic Review, Meta-Analysis, and Proposed Minimum Data Set for Study Level Reporting. Radiology. 2005;237(3):893-904 doi:10.1148/radiol.2373050176

16. Burling D. CT colonography standards. Clin Radiol. 2010;65(6):474-80 doi:10.1016/j.crad.2009.12.003

17. Lung PFC, Burling D, Kallarackel L, et al. Implementation of a new CT colonography service: 5 year experience. Clin Radiol. 2014;69(6):597-605 doi:10.1016/j.crad.2014.01.007

18. Dyde R, Chapman AH, Gale R, Mackintosh A, Tolan DJM. Precautions to be taken by radiologists and radiographers when prescribing hyoscine-N-butylbromide. Clin Radiol. 2008;63(7):793-43 doi:10.1016/j.crad.2008.02.008

19. Boellaard TN, De Haan MC, Venema HW, Stoker J. Colon distension and scan protocol for CT-colonography: An overview. Eur J Radiol. 2013;82(8):1144-58 doi:10.1016/j.ejrad.2011.10.030

20. Båth M, Hansson J. VGC analyzer: A software for statistical analysis of fully crossed multiplereader multiple-case visual grading characteristics studies. Radiat Prot Dosimetry. 2016;169(1-4):46-53 doi:10.1093/rpd/ncv542

21. Hansson J, Månsson LG, Båth M. The validity of using ROC software for analysing visual grading characteristics data: An investigation based on the novel software VGC analyzer. Radiat Prot Dosimetry. 2016;169(1-4):54-9 doi:10.1093/rpd/ncw035

22. Båth $M$, Månsson LG. Visual grading characteristics (VGC) analysis: A non-parametric rankinvariant statistical method for image quality evaluation. Br J Radiol. 2007;80(951):169-176. doi:10.1259/bjr/35012658

23. Båth M. Evaluating imaging systems: Practical applications. Radiat Prot Dosimetry. 2010;139(1-3):26-36 doi:10.1093/rpd/ncqoo7

24. Kim B, Park SH, Hong GS, et al. lohexol versus diatrizoate for fecal/fluid tagging during $C T$ colonography performed with cathartic preparation: comparison of examination quality. Eur Radiol. 2015. doi:10.1007/s00330-014-3568-o 
25. Johnson B, Hinshaw JL, Robbins JB, Pickhardt PJ. Objective and subjective intrapatient comparison of iohexol versus diatrizoate for bowel preparation quality at CT colonography. Am J Roentgenol. 2016;206(6):1202-7 doi:10.2214/AJR.15.15373

26. Kessels RPC. Patients' memory for medical information. J R Soc Med. 2003;96(5):219-22 doi:10.1258/jrsm.96.5.219 